\title{
Active control of outgoing noise fields in rooms
}

Fei Ma, Wen Zhang, and Thushara D. Abhayapala

Citation: The Journal of the Acoustical Society of America 144, 1589 (2018); doi: 10.1121/1.5055217

View online: https://doi.org/10.1121/1.5055217

View Table of Contents: https://asa.scitation.org/toc/jas/144/3

Published by the Acoustical Society of America

\section{ARTICLES YOU MAY BE INTERESTED IN}

Spherical harmonics based generalized image source method for simulating room acoustics

The Journal of the Acoustical Society of America 144, 1381 (2018); https://doi.org/10.1121/1.5053579

Two-dimensional multizone sound field reproduction using a wave-domain method

The Journal of the Acoustical Society of America 144, EL185 (2018); https://doi.org/10.1121/1.5054079

Spherical expansions of sound radiation from resilient and rigid disks with reduced error

The Journal of the Acoustical Society of America 144, 1180 (2018); https://doi.org/10.1121/1.5054010

A deep learning based segregation algorithm to increase speech intelligibility for hearing-impaired listeners in reverberant-noisy conditions

The Journal of the Acoustical Society of America 144, 1627 (2018); https://doi.org/10.1121/1.5055562

Assimilation of mobile phone measurements for noise mapping of a neighborhood

The Journal of the Acoustical Society of America 144, 1279 (2018); https://doi.org/10.1121/1.5052173

Application of the remote microphone method to active noise control in a mobile phone

The Journal of the Acoustical Society of America 143, 2142 (2018); https://doi.org/10.1121/1.5031009 


\title{
Active control of outgoing noise fields in rooms
}

\author{
Fei Ma, ${ }^{1, a)}$ Wen Zhang, ${ }^{2, b)}$ and Thushara D. Abhayapala ${ }^{1}$ \\ ${ }^{1}$ College of Engineering and Computer Science, The Australian National University, Canberra, \\ Australian Capital Territory 0200, Australia \\ ${ }^{2}$ Center of Intelligent Acoustics and Immersive Communications, Northwestern Polytechnical University, \\ $X i$ ' an 710072, Shaanxi, China
}

(Received 19 May 2018; revised 26 August 2018; accepted 30 August 2018; published online 26 September 2018)

\begin{abstract}
Current active noise control systems can cancel noises in a duct effectively. However, they are insufficient for suppressing complex noise fields in time-varying rooms. This paper develops an active noise control system that can cancel tonal noise fields produced by a primary source in a room. The problem of tonal noise field control is formulated as estimating and canceling the outgoing field on a sphere surrounding the primary source. The proposed system limits the energy of the primary source radiating out of the sphere, thereby creating a global quiet zone inside the room. In addition, it removes the need for online secondary path estimation with reduced influence on desired sound fields in the room. A method for estimating the outgoing field on a sphere is presented, together with a wave-domain algorithm for controlling the outgoing field. Simulations and hardware demonstrations show the proposed system can reduce tonal noise fields in a room and over a wide frequency range. (C) 2018 Acoustical Society of America.
\end{abstract}

https://doi.org/10.1121/1.5055217

Pages: 1589-1599

\section{INTRODUCTION}

An active noise control (ANC) system reduces the primary noise by superposing it with the secondary noise at the error sensor. ${ }^{1}$ To deal with non-stationary primary noises, ANC systems use adaptive filters to adjust the secondary source driving signals. In time-varying environments, the secondary path, i.e., the impulse response or the transfer function between the secondary source and the error sensor, needs to be identified online to compensate its influence on the optimization of the adaptive filter coefficients. ${ }^{1}$

Using the least-mean-square (LMS) type algorithms to adjust the secondary source driving signal and the auxiliarynoise-aided method for online secondary path modeling, current ANC systems can control noises in a duct effectively. ${ }^{1}$ However, it is difficult to control noise fields over spatial regions using existing ANC systems.

Earlier work on spatial noise field control problems extended the single-channel LMS-type algorithm into multichannel LMS-type algorithms., ${ }^{2,3}$ The ANC systems based on the multi-channel LMS-type algorithms can reduce the noise pressures around the error sensors. However, these systems have not exploited spatial noise field characteristics ${ }^{2,3}$ and provide little insight into spatial noise field control problems.

Instead of controlling noise pressures at error sensors directly, novel spatial noise field control strategies were developed based on modeling noise fields and manipulating the modeling coefficients of the noise field. Kempton first used the Taylor series to expand the free-space Green

\footnotetext{
${ }^{\text {a)} E l e c t r o n i c ~ m a i l: ~ f e i . m a @ i e e e . o r g ~}$

b) Also at: College of Engineering and Computer Science, The Australian National University, Canberra, Australian Capital Territory 0200, Australia.
}

function, showing that a multipole source array can cancel the far-field sound generated by a monopole source. ${ }^{4}$ Later on, the room mode concept was introduce into ANC systems. ${ }^{5,6}$ The room modes are eigenfunctions of the acoustic wave equation, and conform with the geometry of an empty rectangular room. Simulations demonstrated that the room mode based ANC systems can reduce the noise field globally in a room. ${ }^{5}$ Nonetheless, the implementation of room mode based ANC systems is problematic because the performance of these systems depends on the room damp-ratio parameter, whose estimation is demanding in a timevarying room environment. Recently, spherical harmonics, eigenfunctions of the acoustic wave equation in spherical coordinates, were used to expand the noise fields for local and global noise field control systems. ${ }^{7,8}$ The spherical harmonics are powerful tools for sound field analysis and sound field reproduction, ${ }^{9,10}$ shedding light on spatial noise field control problems. ${ }^{7,8,11,12}$

The main drawback of most existing ANC systems in rooms is that these systems have not explicitly taken additional objects, such as people and desired sound sources, into consideration. The additional objects cause two problems for ANC systems in rooms. First, the movement of additional objects makes the noise field in a room fast-changing. The secondary paths need to be identified online for the ANC systems to work. ${ }^{2,3}$ Nonetheless, it is challenging to identify the secondary paths in rooms accurately online. ${ }^{13}$ Consequently, most existing ANC systems will have performance downgrade in real rooms. Second, the additional objects produce desired sound fields in a room apart from the primary noise field. Existing ANC systems, which have neglected this fact, may disturb the desired sound fields. ${ }^{6}$

In this work, we develop an ANC system that can cancel tonal noise fields in a room without the need for online 
secondary path estimation. Here, the tonal noise means the low frequency (e.g., $[50,500] \mathrm{Hz}$ ) periodic noises generated by rotating machines, and the energy of the tonal noise concentrates at several harmonic frequencies. ${ }^{1} \mathrm{We}$ formulate the problem of tonal noise field control in a room as estimating and canceling the outgoing field produced by the primary source. ${ }^{14} \mathrm{We}$ reduce the primary outgoing field by superposing it with the secondary outgoing field. As the secondary outgoing field is independent of room reverberations, the proposed system removes the need for real-time room reverberation characteristics identification, i.e., online secondary path estimation. By controlling the outgoing field only, the proposed system achieves global noise cancellation performance and reduces its influence on the desired sound fields in the room. This paper also presents a method to estimate the outgoing field on a sphere and a wave-domain algorithm for outgoing field cancellation. Simulations and hardware demonstrations confirm the effectiveness of the proposed system for controlling tonal noise fields in rooms over a wide frequency range.

\section{PROBLEM FORMULATION}

Consider a room with a primary source placed at the point $O$ and marked as $\triangle$, as shown in Fig. 1. A set of secondary sources and error sensors are marked as and $\circ$, respectively. The radius of the sphere $\mathbb{S}_{2}$ is $R$. There are desired sound sources (human) in the room apart from the primary and secondary sources. A secondary path consists of the secondary source ( $(-1))$, the microphone (o), the embedded circuits, and the acoustic path. The acoustic path consists of the outgoing path $\longrightarrow$, and the reverberant path $-\rightarrow$. As shown in Fig. 1, the reverberant path is susceptible to the room environment changes, such as the movement of sound scattering objects, thus is time-varying. Let the noise pressure at a point $(r, \theta, \phi)$ due to the primary source be $P(k, r$, $\theta, \phi)$, where $k=2 \pi f / c$ is the wave number, $f$ is the frequency, $c$ is the speed of sound, $r$ is the radial distance, $\theta$ is the

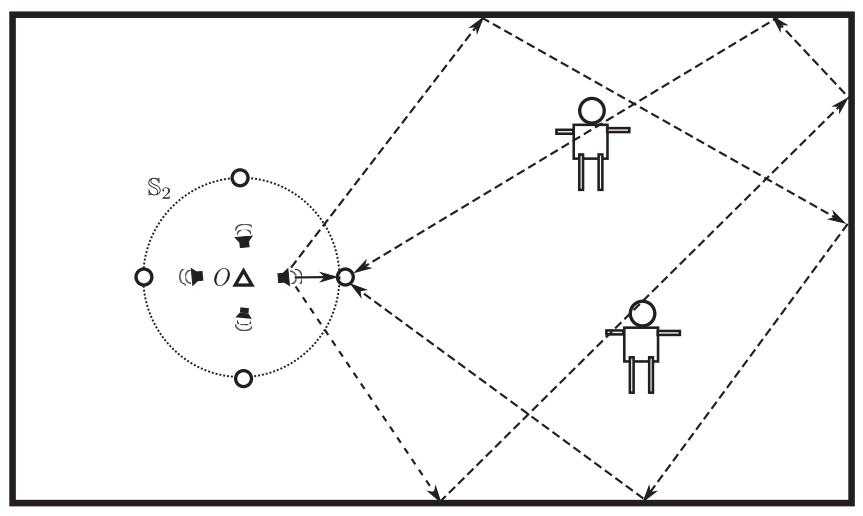

FIG. 1. An example of the proposed ANC system in a room: the primary source is placed at the point $O$ and marked by $\triangle$, secondary sources marked by $\left(\right.$ )), error sensors marked by $\circ$, and $\mathbb{S}_{2}$ denotes a sphere. A secondary path consists of the secondary source ( $(\mathrm{A}))$ ), the microphone (O), the embedded circuits, and the acoustic path. The acoustic path consists of the outgoing path $\longrightarrow$, and the reverberant path $-\rightarrow$. Desired sound sources (human) are expected to be present in the room. elevation, and $\phi$ is the azimuth with respect to a spherical coordinate system whose origin is at the point $O$.

The problem considered in this paper is to cancel the primary noise field $P(k, r, \theta, \phi)$ outside the sphere $\mathbb{S}_{2}$ and inside the room, without online estimation of the secondary paths, and with reduced influences on the desired sound fields in the room.

The primary noise field $P(k, R, \theta, \phi)$ on the sphere $\mathbb{S}_{2}$ surrounding the primary source can be expressed as a spherical harmonic expansion 9,10

$$
P(k, R, \theta, \phi)=\sum_{n=0}^{\infty} \sum_{m=-n}^{n} \alpha_{n m}(k, R) Y_{n m}(\theta, \phi),
$$

where $Y_{n m}(\theta, \phi)$ is the spherical harmonic of order $n$ and degree $m$, and $\alpha_{n m}(k, R)$ are spherical harmonic coefficients evaluated at the radius $R$. In this paper, we omit the time dependence $e^{i 2 \pi f t}$ for notational simplicity, where $i$ is the unit imaginary number and $i^{2}=-1$. Using the spherical harmonic analysis equation, the spherical harmonic coefficients $\alpha_{n m}(k, R)$ are given by ${ }^{9}$

$$
\alpha_{n m}(k, R)=\int_{0}^{2 \pi} \int_{0}^{\pi} P(k, R, \theta, \phi) Y_{n m}^{*}(\theta, \phi) \sin \theta d \theta d \phi,
$$

where ${ }^{*}$ denotes complex conjugation. Hereafter, we abbreviate $(\theta, \phi)$ as a single symbol $\Theta$ to simplify the notation.

The primary noise field $P(k, R, \Theta)$ on the sphere $\mathbb{S}_{2}$ consists of two parts: the primary outgoing field $P^{\mathrm{O}}(k, R, \Theta)$ and the primary incoming field $P^{\mathrm{I}}(k, R, \Theta)^{9,14}$

$$
\begin{aligned}
P(k, R, \Theta)= & \underbrace{\sum_{n=0}^{\infty} \sum_{m=-n}^{n} l_{n m}(k) h_{n}(k R) Y_{n m}(\Theta)}_{P^{\mathrm{O}}(k, R, \Theta)} \\
& +\underbrace{\sum_{n=0}^{\infty} \sum_{m=-n}^{n} \kappa_{n m}(k) j_{n}(k R) Y_{n m}(\Theta)}_{P^{\mathrm{I}}(k, R, \Theta)},
\end{aligned}
$$

where $h_{n}(\cdot)$ is the spherical Hankel function of the second kind with order $n, j_{n}(\cdot)$ is the spherical Bessel function of the first kind with order $n, l_{n m}(k)$ and $\kappa_{n m}(k)$ denote the outgoing and incoming field coefficients, respectively.

Note that the primary outgoing field $P^{\mathrm{O}}(k, R, \Theta)$ consists of the sound generated directly by the primary source and the sound scattered from the primary source surface and its surroundings. ${ }^{15,16}$ The primary incoming field $P^{\mathrm{I}}(k, R$, $\Theta)$, on the other hand, is due to room reverberations. ${ }^{17}$

In this paper, we formulate the problem of controlling the primary noise field $P(k, r, \Theta)$ in the room as estimating and canceling the primary outgoing field $P^{\mathrm{O}}(k, R, \Theta)$ on the sphere $\mathbb{S}_{2}$. By canceling the primary outgoing field $P^{\mathrm{O}}(k, R$, $\Theta)$, we effectively cancel the undesired noise in the entire room. Further, given that the desired sound sources locate outside of the sphere $\mathbb{S}_{2}$, the desired fields produced by them do not contribute to the primary outgoing field $P^{\mathrm{O}}(k, R, \Theta)$ on the sphere $\mathbb{S}_{2}$. Thus, by canceling the primary outgoing 
field $P^{\mathrm{O}}(k, R, \Theta)$ only, the noise cancellation system can reduce its influence on the desired sound fields.

\section{ESTIMATION OF THE OUTGOING FIELD ON A SPHERE}

In this section, we introduce a method to estimate the outgoing field on a sphere. The effectiveness of this method has been confirmed by simulations and experiments, ${ }^{17}$ and we present detailed derivations of this method here for completeness.

\section{A. Pressure and radial particle velocity expansions}

Let the primary noise field on the sphere $\mathbb{S}_{2}$ be sampled by an array of error sensors placed at $\left\{R, \Theta_{q}\right\}_{q=1}^{Q_{\mathrm{e}}}$, as shown in Fig. 1. Using Eq. (3), the primary noise pressures at sampling points $q=1, \ldots, Q_{e}$ can be expressed as ${ }^{9}$

$$
\begin{aligned}
P\left(k, R, \Theta_{q}\right) \approx & \sum_{n=0}^{N_{R}} \sum_{m=-n}^{n} \alpha_{n m}(k, R) Y_{n m}\left(\Theta_{q}\right) \\
= & \sum_{n=0}^{N_{R}} \sum_{m=-n}^{n}\left[l_{n m}(k) h_{n}(k R)\right. \\
& \left.+\kappa_{n m}(k) j_{n}(k R)\right] Y_{n m}\left(\Theta_{q}\right),
\end{aligned}
$$

where both the outgoing and incoming field spherical harmonic expansions are truncated to order $N_{R}=\lceil k \exp (1) R /$ $27 .{ }^{18-21}$ The truncation order $N_{R}$ is determined by the radius of the sphere $\mathbb{S}_{2}$ and the wavenumber $k$, and by the bounds on the spherical Bessel function $j_{n}(k R)$ which also decides the upper limit of $t_{n m}$ in Eq. (4). [Because first, both the incoming field coefficients $\kappa_{n m}(k)$ and the spherical harmonics $Y_{n m}(\cdot)$ are bounded functions; second, the spherical Hankel function $h_{n}(k R)$ has a weaker impact than the same order of the spherical Bessel function $j_{n}(k R) .{ }^{22}$ ]

The radial particle velocities at the sampling points can be expressed as ${ }^{9}$

$$
\begin{aligned}
V\left(k, R, \Theta_{q}\right)= & \sum_{n=0}^{\infty} \sum_{m=-n}^{n} \beta_{n m}(k, R) Y_{n m}\left(\Theta_{q}\right) \\
= & \left.\frac{i}{\rho c k} \frac{\partial P\left(k, r, \Theta_{q}\right)}{\partial r}\right|_{r=R} \\
\approx & \frac{i}{\rho c} \sum_{n=0}^{N_{R}} \sum_{m=-n}^{n}\left[l_{n m}(k) h_{n}^{\prime}(k R)\right. \\
& \left.+\kappa_{n m}(k) j_{n}^{\prime}(k R)\right] Y_{n m}\left(\Theta_{q}\right),
\end{aligned}
$$

where $\beta_{n m}(k, R)$ are the spherical harmonic coefficients of the radial particle velocities $V\left(k, R, \Theta_{q}\right)$ on the sphere $\mathbb{S}_{2}, h_{n}^{\prime}(z)=\partial h_{n}(z) / \partial z$ and $j_{n}^{\prime}(z)=\partial j_{n}(z) / \partial z$ are derivatives of the spherical Hankel function $h_{n}(z)$ and the spherical Bessel function $j_{n}(z)$ with respect to the argument $z$, respectively, and $\rho$ is the density of air.

Theoretically, the spherical harmonic coefficients $\alpha_{n m}(k$, $R)$ and $\beta_{n m}(k, R)$ can be computed using the analytical Eq. (2) if the pressure and radial particle velocity are known over the continuous sphere $\mathbb{S}_{2}{ }^{23}$ However, in practice, only finite samples of the pressure and radial particle velocity are available. Hence, we obtain the spherical harmonic coefficients $\alpha_{n m}(k, R)$ and $\beta_{n m}(k, R)$ through

$$
\begin{aligned}
& \alpha_{n m}(k, R) \approx \sum_{q=1}^{Q_{\mathrm{e}}} \tau_{q} P\left(k, R, \Theta_{q}\right) Y_{n m}^{*}\left(\Theta_{q}\right), \\
& \beta_{n m}(k, R) \approx \sum_{q=1}^{Q_{\mathrm{e}}} \tau_{q} V\left(k, R, \Theta_{q}\right) Y_{n m}^{*}\left(\Theta_{q}\right),
\end{aligned}
$$

where $n \in\left[0, N_{R}\right], m \in[-n, n]$, and $\left\{\tau_{q}\right\}_{q=1}^{Q_{\mathrm{e}}}$ are the sampling weights. ${ }^{23}$ To accurately estimate the spherical harmonic coefficients up to order $N_{R}$, the number of sampling points need to satisfy $Q_{e} \geq\left(N_{R}+1\right)^{2} .{ }^{23}$

\section{B. Estimating the outgoing and incoming field coefficients}

In this section, we estimate the outgoing and incoming field coefficients based on the spherical harmonic coefficients of the pressure and radial particle velocity on the sphere $\mathbb{S}_{2}$.

From the right-hand sides of Eqs. (4) and (5), the outgoing field coefficients $l_{n m}(k)$ and the incoming field coefficients $\kappa_{n m}(k)$ relate with the spherical harmonic coefficients $\alpha_{n m}(k, R)$ and $\beta_{n m}(k, R)$ through

$$
\begin{aligned}
& \alpha_{n m}(k, R)=i_{n m}(k) h_{n}(k R)+\kappa_{n m}(k) j_{n}(k R), \\
& -i \rho c \beta_{n m}(k, R)=i_{n m}(k) h_{n}^{\prime}(k R)+\kappa_{n m}(k) j_{n}^{\prime}(k R) .
\end{aligned}
$$

Using Eq. (7), we obtain the outgoing field coefficients $\imath_{n m}(k)$ as

$$
\imath_{n m}(k)=\frac{\alpha_{n m}(k, R) j_{n}^{\prime}(k R)+i \rho c \beta_{n m}(k R) j_{n}(k R)}{h_{n}(k R) j_{n}^{\prime}(k R)-h_{n}^{\prime}(k R) j_{n}(k R)} .
$$

Substitution of the Wronskian relation ${ }^{9}$

$$
h_{n}(k R) j_{n}^{\prime}(k R)-h_{n}^{\prime}(k R) j_{n}(k R)=\frac{-i}{k^{2} R^{2}},
$$

in Eq. (8), produces

$$
\imath_{n m}(k)=\Delta\left[-i \alpha_{n m}(k, R) j_{n}^{\prime}(k R)+\rho c \beta_{n m}(k, R) j_{n}(k R)\right],
$$

where $\Delta=k^{2} R^{2}$. Similarly, using Eqs. (6), (7), and (9), we obtain the incoming field coefficients $\kappa_{n m}(k)$ as

$$
\kappa_{n m}(k)=\Delta\left[i \alpha_{n m}(k, R) h_{n}^{\prime}(k R)-\rho c \beta_{n m}(k, R) h_{n}(k R)\right] .
$$

Substituting Eqs. (10) and (11) into corresponding parts of Eq. (3) yields estimations of the primary outgoing field and the primary incoming field, respectively.

We have the following comments on Eq. (10):

(1) The outgoing field is uniquely determined by the outgoing field coefficients $\imath_{n m}(k)$ and can be controlled by manipulating the coefficients $l_{n m}(k)$. 
(2) The outgoing field coefficients $l_{n m}(k)$ are radial independent. The outgoing field on one sphere of radius $R_{1}$ can be projected to another sphere of radius $R_{2}$, where $R_{2}>R_{1} .{ }^{9}$ By reducing the outgoing field on a small sphere surrounding the primary source, we essentially reduce the outgoing field on all outer spheres with larger radii. This fact enables the proposed ANC system to cancel noises globally in a room.

\section{SPATIAL NOISE FIELD CANCELLATION}

In this section, we develop a wave-domain mode matching method to compute the secondary source driving signals needed for outgoing field cancellation, and preset the implementation of the noise cancellation process in Sec. IV A.

The idea is to use the secondary outgoing field to destructively interfere with the primary outgoing field. We place $Q_{\text {s }}$ point sources between the primary source and the sphere $\mathbb{S}_{2}$ as the secondary sources as shown in Fig. 1. The secondary outgoing field $S^{\mathrm{O}}(k, R, \Theta)$ on the sphere $\mathbb{S}_{2}$ is represented as

$$
S^{\mathrm{O}}(k, R, \Theta) \approx \sum_{n=0}^{N_{R}} \sum_{m=-n}^{n} \chi_{n m}(k) h_{n}(k R) Y_{n m}(\Theta),
$$

where we also truncate the secondary outgoing field $S^{\mathrm{O}}(k, R$, $\Theta)$ to order $N_{R}$, and the expressions of the secondary outgoing field coefficients $\chi_{n m}(k)$ are $^{9}$

$$
\chi_{n m}(k)=-i k \sum_{q=1}^{Q_{\mathrm{s}}} w_{q}(k) j_{n}\left(k r_{q}\right) Y_{n m}^{*}\left(\Theta_{q}\right),
$$

$n \in\left[0, N_{R}\right], m \in[-n, n],\left(r_{q}, \Theta_{q}\right)$, and $w_{q}(k)$ are the spherical coordinates and the driving signal of the $q$ th secondary source, respectively.

The matrix form of Eq. (13) is

$$
\boldsymbol{\chi}(k)=\boldsymbol{G}(k) \boldsymbol{w}(k),
$$

where $\chi(k)=\left[\chi_{00}(k), \chi_{1-1}(k), \ldots, \chi_{N_{R} N_{R}}(k)\right]^{T}\left(^{T}\right.$, is the transpose operator) is a $\left(N_{R}+1\right)^{2} \times 1$ vector, $\boldsymbol{w}(k)=\left[w_{1}(k)\right.$, $\left.w_{2}(k), . ., w_{Q_{\mathrm{s}}}(k)\right]^{T}$ is a $Q_{\mathrm{s}} \times 1$ vector of the secondary source driving signals, and $\boldsymbol{G}(k)$ is a $\left(N_{R}+1\right)^{2} \times Q_{\mathrm{s}}$ matrix

$$
\boldsymbol{G}(k)=\left[\begin{array}{cccc}
g_{00}(1) & g_{00}(2) & \ldots & g_{00}\left(Q_{\mathrm{s}}\right) \\
g_{1-1}(1) & g_{1-1}(2) & \ldots & g_{1-1}\left(Q_{\mathrm{s}}\right) \\
\vdots & \vdots & \ddots & \vdots \\
g_{N_{R} N_{R}}(1) & g_{N_{R} N_{R}}(2) & \ldots & g_{N_{R} N_{R}}\left(Q_{\mathrm{s}}\right)
\end{array}\right]
$$

with entries $g_{n m}(q)=-i k j_{n}\left(k r_{q}\right) Y_{n m}^{*}\left(\Theta_{q}\right)$.

The cancellation of the outgoing field requires

$$
S^{\mathrm{O}}(k, R, \Theta)+P^{\mathrm{O}}(k, R, \Theta)=0,
$$

where $S^{\mathrm{O}}(k, R, \Theta)$ and $P^{\mathrm{O}}(k, R, \Theta)$ are given by Eqs. (12) and (3), respectively. Using the spherical harmonics expansion of each term, Eq. (16) can be expressed as

$$
\chi_{n m}(k)+\imath_{n m}(k)=0, \quad n \in\left[0, N_{R}\right], m \in[-n, n] .
$$

Based on Eq. (14), Eq. (17) can be expressed as a matrix equation

$$
\boldsymbol{G}(k) \boldsymbol{w}(k)=\boldsymbol{\psi}(k),
$$

where

$$
\psi(k)=-\left[l_{00}(k), l_{1-1}(k), \ldots, l_{N_{R} N_{R}}(k)\right]^{T},
$$

is a $\left(N_{R}+1\right)^{2} \times 1$ vector.

We solve Eq. (18) as a least square problem to obtain the secondary source driving signals needed for outgoing field cancellation

$$
\boldsymbol{w}(k)=\boldsymbol{G}^{+}(k) \boldsymbol{\psi}(k),
$$

where $\boldsymbol{G}^{+}(k)$ is the pseudo-inverse of matrix $\boldsymbol{G}(k)$.

The matrix $G(k)$ characterizes the combined transfer function of the secondary source, the error microphone, the embedded circuits, and the outgoing path, excluding the transfer function of the time-varying reverberant path. The matrix $G(k)$ is relatively stationary and can be estimated offline. Therefore, the proposed ANC system does not need to estimate the secondary path transfer function online, or in real time.

Note that using the sound field separation method both the pressure, the radial particle velocity, and the sound intensity associated with the outgoing field can be estimated. More advanced control strategies, such as the active sound intensity control, ${ }^{24,25}$ can be applied to further improve the performance of the proposed ANC system. This will be one of our future works.

\section{A. Implementation of the proposed ANC system}

In this section, we provide details that facilitate the implementation of the proposed ANC system.

We provide the signal flow diagram of the proposed ANC system in Fig. 2. In the system, we first transform the pressure $\left\{P\left(t, R, \Theta_{q}\right)\right\}_{q=1}^{Q_{\mathrm{e}}}$ and radial particle velocity $\left\{V\left(t, R, \Theta_{q}\right)\right\}_{q=1}^{Q_{\mathrm{e}}}$ measurements of the error sensors into time-frequency domain through a Short-Time Fourier Transform (STFT). ${ }^{26}$ Then we decompose the time-frequency domain pressure and radial particle velocity into corresponding spherical harmonic coefficients $\left\{\alpha_{n m}(t, k, R), \beta_{n m}(t, k, R)\right\}_{n m=00}^{N_{R} N_{R}}$ through Eq. (6). Next we obtain the outgoing field coefficients $\left\{l_{n m}(t, k)\right\}_{n m=00}^{N_{R} N_{R}}$ through Eq. (10), and calculate the time-frequency domain secondary source driving signals $\left\{w_{q}(t, k)\right\}_{q=1}^{Q_{\mathrm{s}}}$ using Eq. (19). Last we transform $\left\{w_{q}(t, k)\right\}_{q=1}^{Q_{\mathrm{s}}}$ into time-domain secondary source driving signal $\left\{w_{q}(t)\right\}_{q=1}^{Q_{\mathrm{s}}}$ through an inverse STFT.

In practical implementations of the proposed ANC system, the primary source is most likely to be attached to a surface. We arrange the error sensors on an upper semi-sphere surrounding the primary source as in examples shown in Secs. $\mathrm{V}$ and VI. At low frequency range, the reflection from typical surfaces, such as painted concrete or glass, are high. ${ }^{27}$ The 


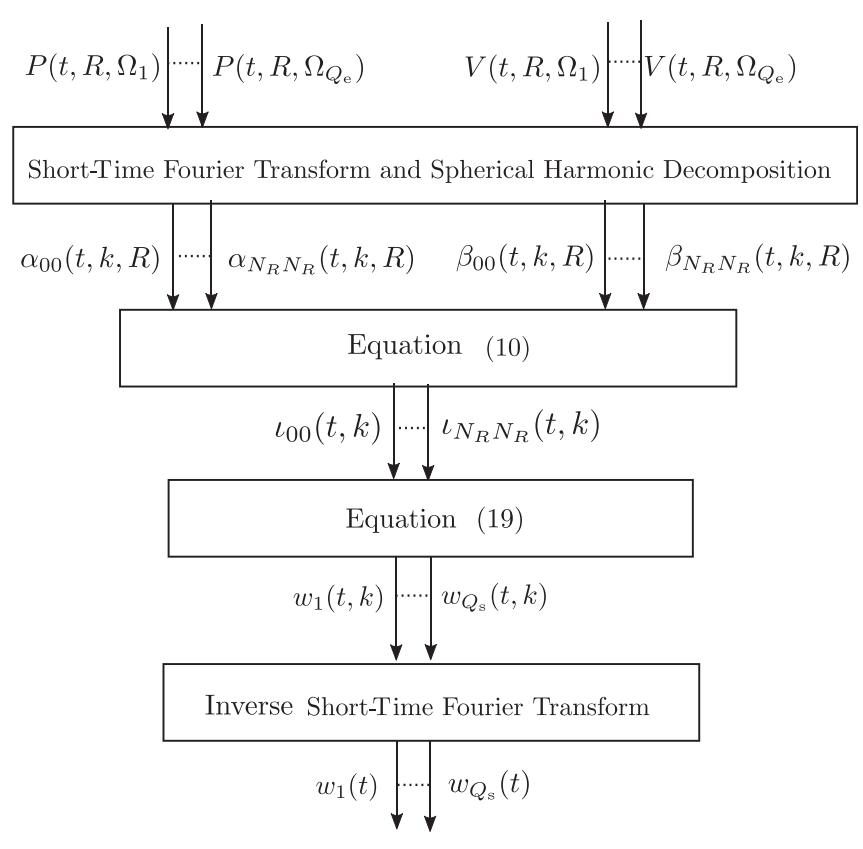

FIG. 2. Signal flow diagram of the proposed ANC system.

measured sound field can be regarded as symmetric with respect to the floor, and can be duplicated to the lower semisphere to use the full spherical harmonic expansion. ${ }^{17}$

\section{SIMULATIONS}

Simulations in this section illustrate the effectiveness of the sound field separation method and the proposed ANC system in a room.

\section{A. Simulation settings}

The simulation environment is a rectangular room of size $4 \mathrm{~m} \times 5 \mathrm{~m} \times 3 \mathrm{~m}$ as shown in Fig. 3. There is a primary source $(\triangle)$ on the floor, three secondary sources $(\triangle(\nabla))$ around the primary source, a number of error sensors ( $(\circ)$ on the upper semi-sphere $\mathbb{S}_{2}$ of radius $R=0.5 \mathrm{~m}$, and a desired sound source $(\bullet)$. We duplicate the sound field measured on the upper semi-sphere to the lower semi-sphere. ${ }^{17} \mathrm{We}$ set up a Cartesian coordinate system and a spherical coordinate system based on the primary source center $O$. One corner of the room locates at $X=(-1.5,-2,0) \mathrm{m}$ with respect to the point $O$. The sampling frequency is $f_{s}=48000 \mathrm{~Hz}$, the speed of sound is $c=343 \mathrm{~m} / \mathrm{s}$, and air density is $\rho=1.225 \mathrm{~m}^{3} / \mathrm{kg}$. The room transfer functions (including the radial particle velocity responses) are simulated using the image source method. ${ }^{28}$ The reflection coefficients of the floor, the ceiling, and walls are all $\gamma=0.995$, and in total 5832 image sources are considered. We add background noise to the error sensor measurements, and the signal power to background noise power ratio is $40 \mathrm{~dB}$. The simulation results are from the average of 100 independent runs. We use the settings in this paragraph for all simulations unless otherwise stated.

\section{B. Sound field separation}

In this simulation, we let the primary source produce a unit-amplitude tonal wave of $200 \mathrm{~Hz}$. The real parts of the outgoing field $\Re\left\{P^{\mathrm{O}}(k, R, \Theta)\right\}$, the reverberation field $\Re\{P(k, R, \Theta)\}$, the estimated outgoing field $\Re\left\{\hat{P}^{\mathrm{O}}(k, R, \Theta)\right\}$, and the field estimation error $\Re\left\{P^{\text {err }}(k, R, \Theta)\right\}=\Re\left\{P^{\mathrm{O}}(k\right.$, $\left.R, \Theta)-\hat{P}^{\mathrm{O}}(k, R, \Theta)\right\}$ on the upper semi-sphere $\mathbb{S}_{2}$ are presented in Figs. 4(a), 4(b), 4(c), and 4(d), respectively. Here, $\Re(\cdot)$ is the real value operator. The imaginary parts of these four fields show similar trends, thus are not shown for brevity. We obtain the outgoing field $P^{\mathrm{O}}(k, R, \Theta)$ and the reverberation field $P(k, R, \Theta)$ by multiplying the primary source strength with the half-space Green function and the room transfer function, respectively. ${ }^{17}$ We place four error sensors on the upper semi-sphere $\mathbb{S}_{2}$ according to the first order Gauss sampling scheme, ${ }^{23}$ and obtain the estimated outgoing field $\hat{P}^{\mathrm{O}}(k, R, \Theta)$ by the sound field separation method. The truncation order of sound field on the upper semi-sphere is $N_{R}=1$. In Fig. 4, the outgoing field $P^{\mathrm{O}}(k, R, \Theta)$ is equal over the semi-sphere $\mathbb{S}_{2}$ as the primary source locates at the center. The reverberation field $P(k, R, \Theta)$ distributes unequally on the semi-sphere $\mathbb{S}_{2}$ due to wall reflections. The estimated outgoing field $\hat{P}^{\mathrm{O}}(k, R, \Theta)$ approximates the outgoing field $P^{\mathrm{O}}(k, R$, $\Theta)$, and the field estimation error $P^{\operatorname{err}}(k, R, \Theta)$ is small over the semi-sphere $\mathbb{S}_{2}$.

In the second simulation, we examine the field estimation error as a function of frequency. Define the normalized estimation error as

$$
\sigma(k)=10 \log _{10} \frac{\sum_{v=1}^{L}\left\|P^{\mathrm{O}}\left(k, R, \Theta_{v}\right)-\hat{P}^{\mathrm{O}}\left(k, R, \Theta_{v}\right)\right\|^{2}}{\sum_{v=1}^{\Pi}\left\|P^{\mathrm{O}}\left(k, R, \Theta_{v}\right)\right\|^{2}},
$$

where $\|\cdot\|$ is the 2-norm, the sound fields are sampled at $L=100 \times 400$ equal-angle points on the upper semi-sphere, and $\left(R, \Theta_{v}\right)$ are the spherical coordinates of the $v$ th sampling point. ${ }^{23}$ Denote $\sigma_{1}(k), \sigma_{2}(k)$, and $\sigma_{3}(k)$ as the normalized estimation errors when the sound field separation method is realized by the first, second, and third order Gauss sampling scheme, i.e., 4, 9, and 16 error sensors on the upper semisphere $\mathbb{S}_{2}$, respectively. In computations of the normalized

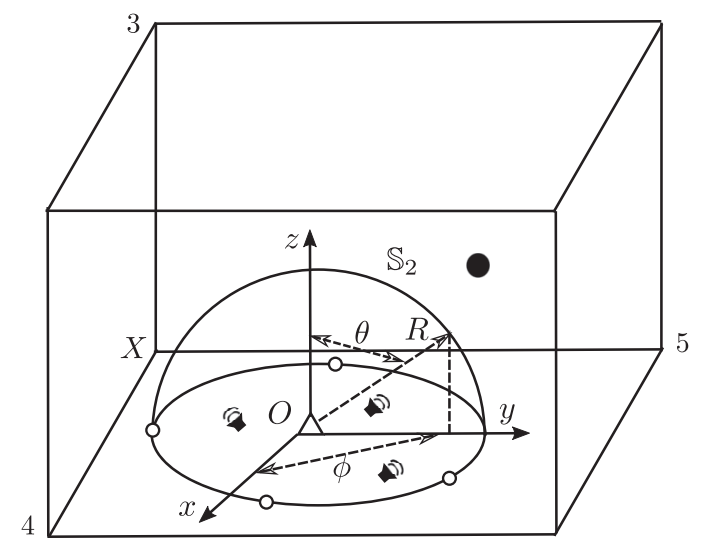

FIG. 3. The simulation environment: The primary source is placed at a point $O$ and marked by $\triangle$, secondary sources marked by $\triangle$ )), error sensors marked by $\circ, \mathbb{S}_{2}$ denotes a semi-sphere enclosing the system, and $\bullet$ denotes a desired sound source. 


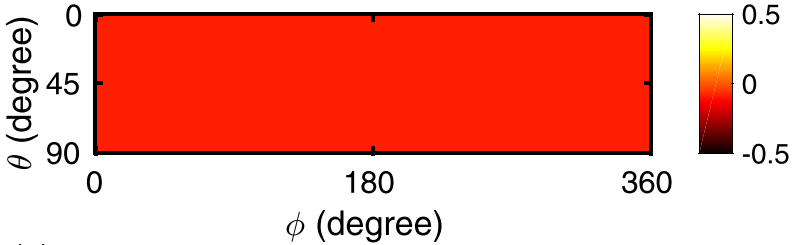

(a)

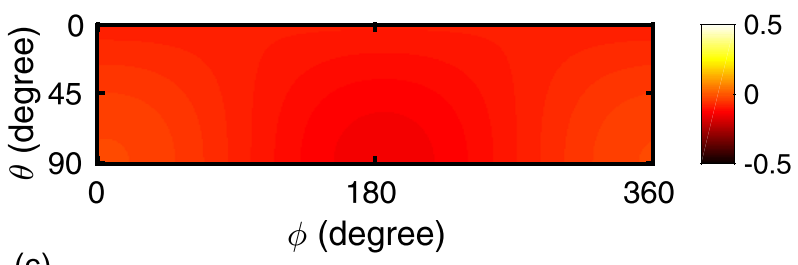

(c)

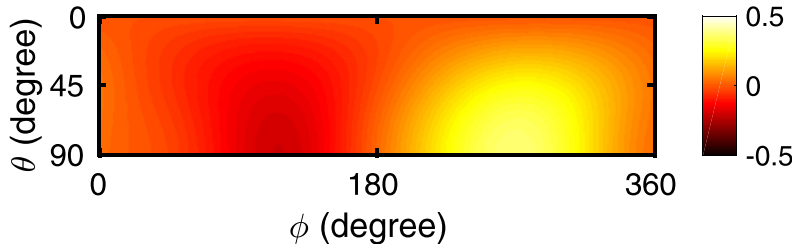

(b)

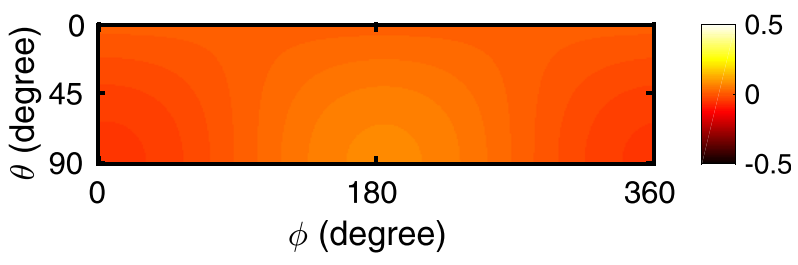

(d)

FIG. 4. (Color online) Sound field separation on the semi-sphere $\mathbb{S}_{2}$ : The real parts of (a) the outgoing field $\Re\left\{P^{\mathrm{O}}(k, R, \Theta)\right\}$, (b) the reverberation field $\Re\{P(k, R, \Theta)\}$, (c) the estimated outgoing field $\Re\left\{\hat{P}^{\mathrm{O}}(k, R, \Theta)\right\}$, and (d) the field estimation error $\Re\left\{P^{\operatorname{err}}(k, R, \Theta)\right\}$.

errors $\sigma_{1}(k), \sigma_{2}(k)$, and $\sigma_{3}(k)$, we choose the truncation orders for the sound field on the upper semi-sphere $\mathbb{S}_{2}$ as $N_{R}=1,2,3$, respectively. We depict $\sigma_{1}(k), \sigma_{2}(k)$, and $\sigma_{3}(k)$ as functions of frequency $f$ in Fig. 5. As shown in Fig. 5, using the first, second, and third order Gauss sampling scheme, we are able to accurately $\left[\sigma_{q}(k)<-20 \mathrm{~dB}, q=1,2\right.$, 3] estimate the outgoing field on the semi-sphere $\mathbb{S}_{2}$ up to 200, 300, and $400 \mathrm{~Hz}$, respectively. This simulation result shows that, by realizing the sound field separation method with an appropriate Gauss sampling scheme, the estimated outgoing field agrees well with the outgoing field over a wide frequency range.

Next we examine the normalized estimation error as a function of the reflection coefficient. We keep the reflection coefficient of the floor constant as $\gamma=0.995$, and change the reflection coefficients $\gamma$ of the ceiling and walls from 0.5 to 0.995 to simulate the variation from a weak reverberant room to a strong reverberant room. We place nine error sensors on the semi-sphere $\mathbb{S}_{2}$ according to the second order Gauss sampling scheme, and choose the truncation order of sound field on the upper semi-sphere as $N_{R}=2$. In Fig. 6, the normalized estimation errors at frequencies 200, 300, and $400 \mathrm{~Hz}$ are labeled as $\sigma_{200}, \sigma_{300}$, and $\sigma_{400}$, respectively. As

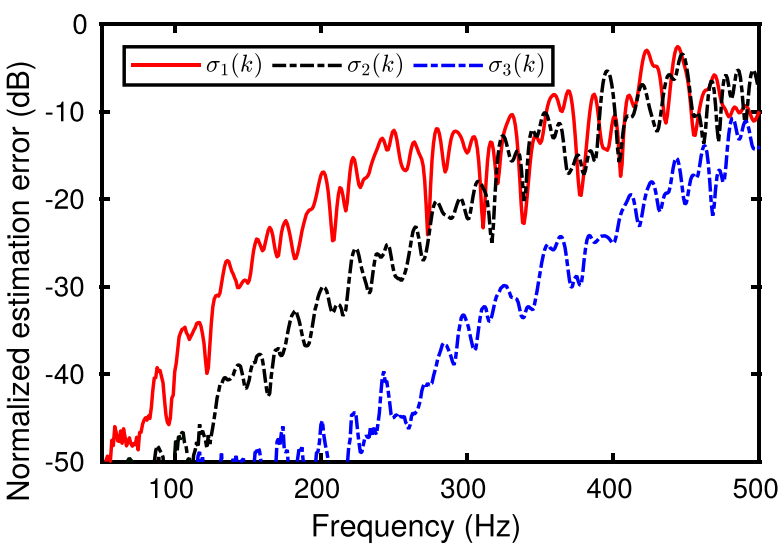

FIG. 5. (Color online) Sound field separation error as a function of frequency: The normalized estimation errors [Eq. (20)] using the first, second, and third order Gauss sampling scheme for sound field separation are labeled as $\sigma_{1}(k), \sigma_{2}(k)$, and $\sigma_{3}(k)$, respectively. shown in Fig. 6, the normalized estimation error increases along with the reflection coefficients.

\section{Comparisons with a classical ANC system}

In this section, we compare the performance of the proposed ANC system with a classical multi-channel ANC system. $^{29}$

In the first simulation, we let the primary source produce a unit-amplitude tonal wave of $230 \mathrm{~Hz}$. Three secondary sources are located at $(0.22,0.0,0.1) \mathrm{m},(-0.11,0.19,0.1)$ $\mathrm{m}$, and $(-0.12,-0.2,0.1) \mathrm{m}$, respectively. In the proposed system, we design the secondary source driving signals to cancel the outgoing field on the upper semi-sphere $\mathbb{S}_{2}$ based on Eq. (19). We obtain the outgoing field coefficients by the sound field separation method, which is realized by four error sensors placed on the upper semi-sphere $\mathbb{S}_{2}$ according to the first order Gauss sampling scheme. ${ }^{30}$ The sound field truncation order is $N_{R}=1$. In the classical system, we calculate the secondary source driving signals using Eq. (12.2.4) from Nelson and Elliott. ${ }^{29}$ Rather than canceling the outgoing field as in the proposed system, the classical system tries to control the noise pressures at $(-1.4,-1.9,0.1) \mathrm{m},(2.4$,

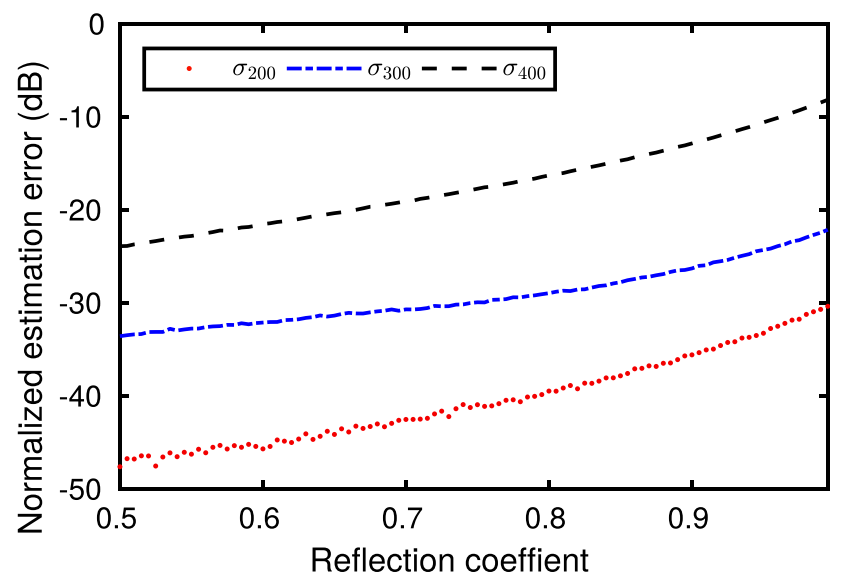

FIG. 6. (Color online) Sound field separation error as a function of the reflection coefficient: The normalized estimation errors at frequencies 200, 300 , and $400 \mathrm{~Hz}$ are labeled as $\sigma_{200}, \sigma_{300}$, and $\sigma_{400}$, respectively. 
$-1.9,0.1) \mathrm{m},(2.4,2.9,0.1) \mathrm{m},(-1.4,2.9,0.1) \mathrm{m},(-1.4$, $-1.9,2.9) \mathrm{m},(2.4,-1.9,2.9) \mathrm{m},(2.4,2.9,2.9) \mathrm{m}$, and $(-1.4,2.9,2.9) \mathrm{m}$ (the eight corners of the room).

We present the results of the first simulation in Fig. 7. Figure 7 (a) depicts the primary field energy $\|P(k, \boldsymbol{x})\|^{2}$. Figure $7(\mathrm{~b})$ depicts the residual field energy $\|E(k, \boldsymbol{x})\|^{2}$ $=\|P(k, \boldsymbol{x})+S(k, \boldsymbol{x})\|^{2}$ in the proposed system, where $S(k, \boldsymbol{x})$ is the secondary field generated by the secondary sources. Here $\boldsymbol{x}=(x, y, z)$ are Cartesian coordinates of 120000 sampling points, which are arranged uniformly on the $x-z$ plane. The residual field energy in the classical system is depicted in Fig. 7(c). In Fig. 7, the semi-circle denotes the semisphere $\mathbb{S}_{2}$. As shown in Fig. 7, in both the proposed and classical system, the residual field energy $\|E(k, x)\|^{2}$ is about $20 \mathrm{~dB}$ less than the primary field energy $\|P(k, \boldsymbol{x})\|^{2}$ on the $x-z$ plane and outside of the semi-sphere.

In the second simulation, we add a desired unit-amplitude point source (marked as $\bullet$ in Figs. 3 and 8 ) into the room at $(1.5,0.0,1.8) \mathrm{m}$. The desired point source also generates a tonal wave of $230 \mathrm{~Hz}$. Other simulating settings are the same as in the first simulation. The desired field energy $\|D(k, \boldsymbol{x})\|^{2}$ and the total field energy $\|T(k, \boldsymbol{x})\|^{2}=\|P(k, \boldsymbol{x})+D(k, \boldsymbol{x})\|^{2}$ on the $x-z$ plane are depicted in Figs. 8(a) and 8(b), respectively. The residual field energy $\|E(k, \boldsymbol{x})\|^{2}=\| P(k, \boldsymbol{x})$ $+D(k, \boldsymbol{x})+S(k, \boldsymbol{x}) \|^{2}$ in the proposed and classical system is given by Figs. 8(c) and 8(d), respectively. In Fig. 8, the semicircle also denotes the semi-sphere $\mathbb{S}_{2}$.

A comparison of Figs. 8(a), 8(b), and 8(c) reveals that the proposed system reduces the total field energy $\|T(k, \boldsymbol{x})\|^{2}$, whilst the residual field energy approximates the desired field energy, i.e., $\|E(k, \boldsymbol{x})\|^{2} \approx\|D(k, \boldsymbol{x})\|^{2}$, on the $x-z$ $\mathrm{m}$ plane and outside of the semi-sphere. A comparison of Figs. 8(a), 8(b), and 8(d) reveals that the classical system reduces the total field energy $\|T(k, x)\|^{2}$, but the residual field energy differs from the desired field energy, i.e., $\|E(k, \boldsymbol{x})\|^{2} \nsucceq\|D(k, \boldsymbol{x})\|^{2}$, over the $x-z$ plane.
The simulation results demonstrate the advantage of the proposed system compared with the classical system. By arranging the noise control system inside a small semisphere and controlling the outgoing field only, the proposed system can reduce its influence on the desired sound fields in the room. The classical system, on the other hand, may cancel the desired sound fields together with the primary noise field.

\section{Noise cancellation over a wide frequency range}

In this section, we conduct noise cancellation over a wide frequency range using the proposed system, without considering desired sound sources. We let the primary source produce a unit-amplitude tonal wave at a single frequency $f(f \in[50,500] \mathrm{Hz})$, and use three secondary sources to control the primary noise field in two cases:

(1) The three secondary sources are located at $(0.22,0.0$, $0.1) \mathrm{m},(-0.11,0.19,0.1) \mathrm{m}$, and $(-0.12,-0.2,0.1) \mathrm{m}$, respectively. The distances from the secondary sources to the origin are $0.24,0.25$, and $0.26 \mathrm{~m}$, respectively.

(2) The three secondary sources are located at $(0.173,0.0$, $0.1) \mathrm{m},(-0.075,0.13,0.1) \mathrm{m}$, and $(-0.081,-0.14,0.1)$ $\mathrm{m}$, respectively. The distances from the secondary sources to the origin are $0.2,0.18$, and $0.19 \mathrm{~m}$, respectively.

Other simulation settings are the same as in the first simulation of Sec. V C.

The performances of the proposed ANC system is characterized by noise field energy reduction in the room,

$$
\xi(k)=10 \log _{10} \frac{\sum_{v=1}^{L}\left\|P\left(k, \boldsymbol{x}_{v}\right)\right\|^{2}}{\sum_{v=1}^{L}\left\|E\left(k, \boldsymbol{x}_{v}\right)\right\|^{2}},
$$

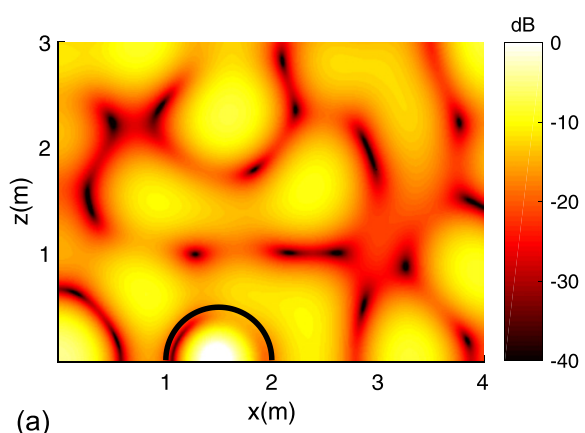

(a)

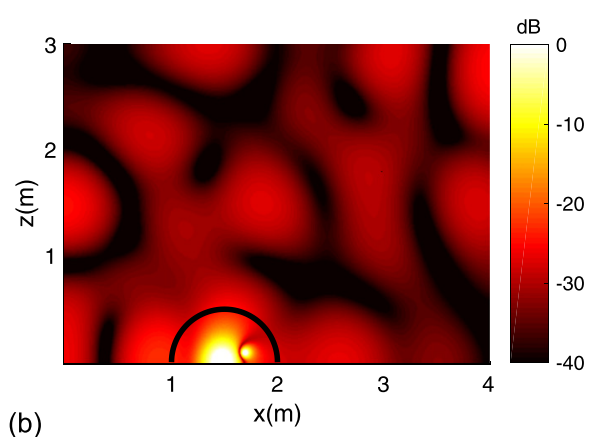

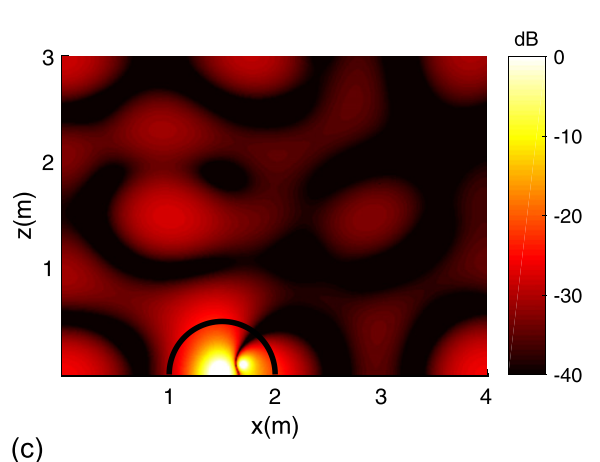

(c)

(b)
FIG. 7. (Color online) Comparison between the proposed and classical system without any desired sound sources: (a) the primary field energy $\|P(k, x)\|^{2}$, the residual field energy $\|E(k, x)\|^{2}$ in the (b) proposed and (c) classical system. 


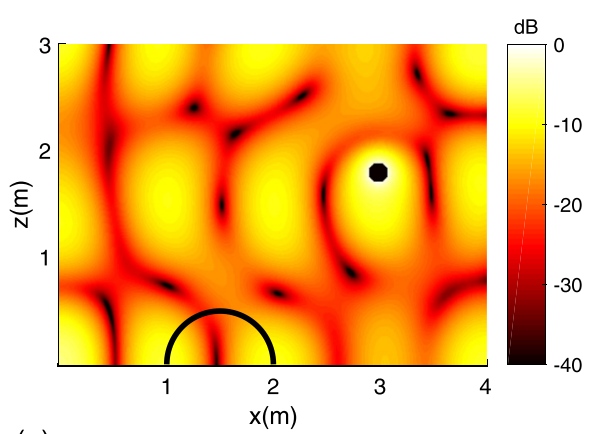

(a)

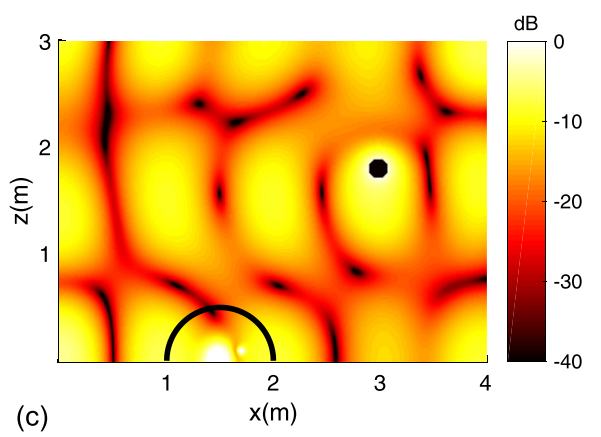

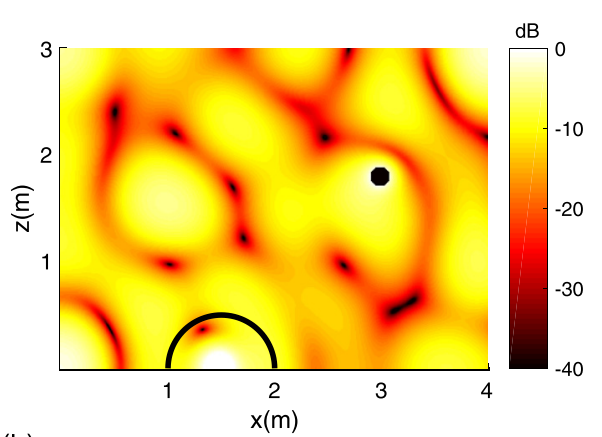

(b)

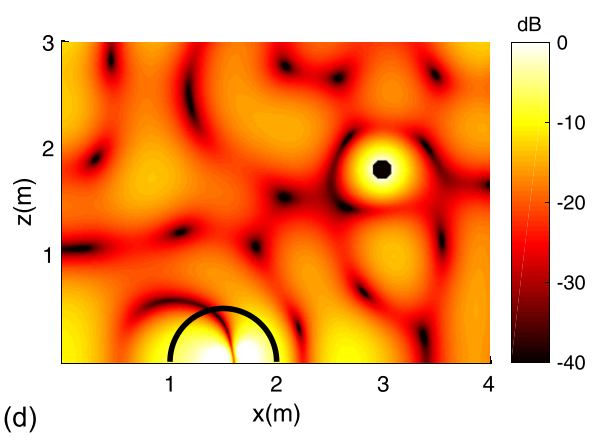

FIG. 8. (Color online) Comparison between the proposed and the classical system with a desired sound source: (a) the desired field energy $\|D(k, x)\|^{2}$, (b) the total field energy $\|T(k, x)\|^{2}$, the residual field energy $\|E(k, \boldsymbol{x})\|^{2}$ in the (c) proposed and (d) classical system. where $P\left(k, \boldsymbol{x}_{v}\right)$ is the primary noise pressure, $E\left(k, \boldsymbol{x}_{v}\right)$ is the residual noise pressure, $L=60000$ is the number of sampling points, and $\boldsymbol{x}_{\nu}=\left(x_{v}, y_{v}, z_{v}\right)$ denotes the $v$ th sampling point position. Here, $x_{v}=-1.4,-1.3, \ldots, 2.5 \mathrm{~m}, y_{v}=-1.9$, $-1.8, \ldots, 3.0 \mathrm{~m}$, and $z_{v}=0.1,0.2, \ldots, 3.0 \mathrm{~m}$. The sampling points inside of the semi-sphere $\mathbb{S}_{2}$ are excluded in summations of Eq. (21). The simulation results of the first and second cases are presented in Fig. 9 as $\xi_{1}(k)$ and $\xi_{2}(k)$, respectively.

As shown in Fig. 9, in the first case, the proposed system reduces the noise field energy in the room by more than $10 \mathrm{~dB}$ over the frequency range $[50,400] \mathrm{Hz}$. In the second case, where the secondary sources are placed closer to the primary source, the proposed system reduces the noise field energy in the room by more than $10 \mathrm{~dB}$ over the frequency range $[50,500] \mathrm{Hz}$.

\section{HARDWARE DEMONSTRATION}

We implement the proposed ANC system in our laboratory to validate its effectiveness for reducing real noises.

The demonstration environment is a room of size about $(3.6,6.7,2.8) \mathrm{m}$ as shown in Fig. 10. There is a thin layer of

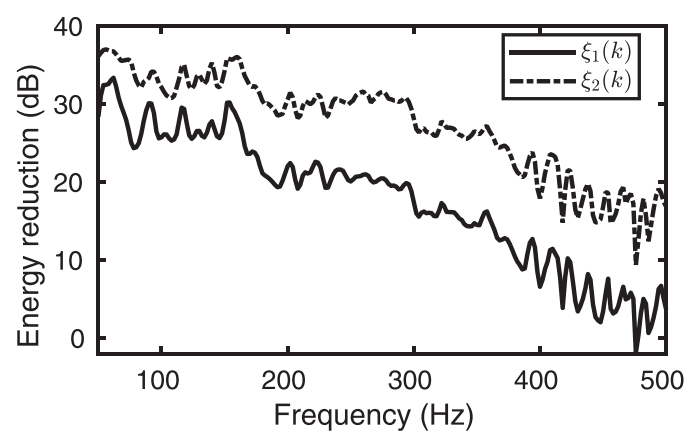

FIG. 9. Noise cancellation over a wide frequency range: Noise field energy reduction achieved by the proposed ANC system over the frequency range $[50,500] \mathrm{Hz}$ in the first case $\xi_{1}(k)$ and in the second case $\xi_{2}(k)$. carpet on the concrete floor, and many objects in the room. The room reverberation time is $T_{60} \approx 1.5 \mathrm{~s}$. We have four loudspeakers on the plywood. The loudspeaker at the center is the primary source, and the other three loudspeakers are secondary sources. All loudspeakers are approximately circular cylinders, with each having a height of $0.11 \mathrm{~m}$ and a radius of $0.055 \mathrm{~m}$. The loudspeaker drivers are Dayton Audio ND90-8 from Dayton Audio (USA). We set up a spherical coordinate system with the origin at the center of the bottom of the primary source. The distances from the secondary sources to the origin are about $0.2 \mathrm{~m}$, and the distances between secondary sources are the same. We have four pairs of microphones labeled as 1, 2; 3, 4; 5, 6; 7, 8 as shown in Fig. 10. The inner four microphones (labeled as 1, 3 , 5, and 7) are placed on a semi-sphere of radius $R_{1}=0.45 \mathrm{~m}$ according to the first order Gauss sampling scheme. The distance between these inner four microphones to the ground is about $0.2 \mathrm{~m}$. The outer four microphones (labeled as 2, 4, 6, and 8) are placed on a semi-sphere of radius $R_{2}=0.55 \mathrm{~m}$ according the first order Gauss sampling scheme. The distance between these outer four microphones to the ground is about $0.25 \mathrm{~m}$. These four microphone pairs are the error sensors, providing pressure and velocity information on a semi-sphere of radius $R=0.5 \mathrm{~m} .{ }^{17}$ We have eight more microphones labeled as $9,10, \ldots, 16$ in the room as shown in Fig. 10. These eight microphones monitor the noise pressure levels in the room but are not part of the ANC system. All 16 microphones are Dayton Audio EMM-6 precision electric condenser microphones from Dayton Audio (USA), and have been calibrated up to $1000 \mathrm{~Hz}$. The analogy-to-digital converter is Behringer Ultragain ADA 8200 from Behringer (Germany), and the digital-to-analogy converter is Rednet 2 from Focusrite Audio Engineering Ltd. (England). The precision of both ADA8200 and Rednet 2 is 24 bit. We used a desktop computer to process the signals. The sampling frequency is $f_{\mathrm{s}}=48000 \mathrm{~Hz}$ and the speed of 


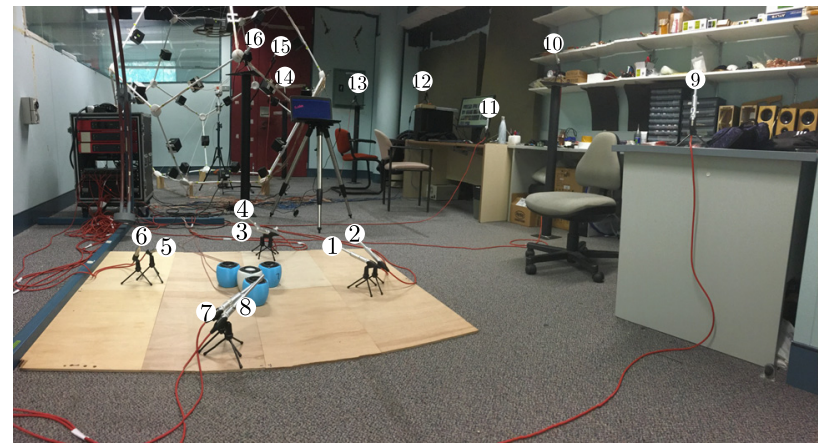

(a)

(b)

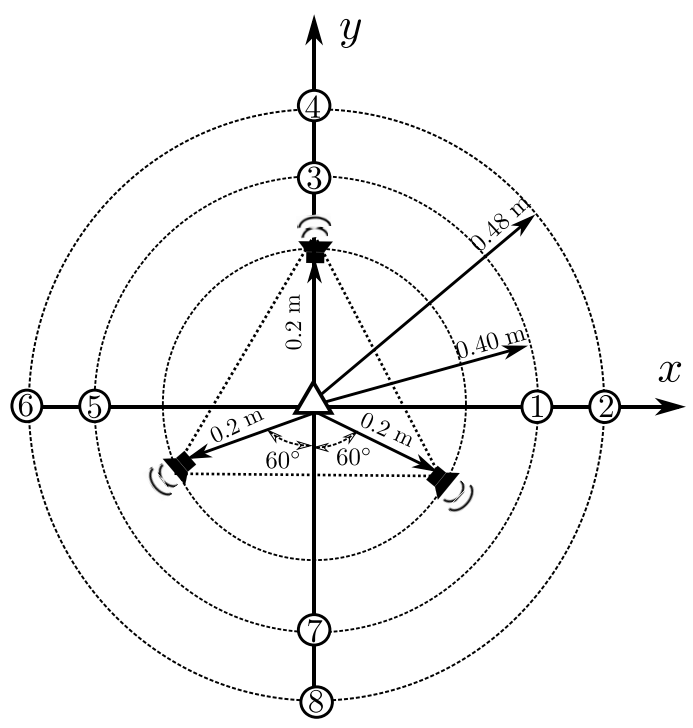

FIG. 10. (Color online) The hardware demonstration environment. (a) An overview of the sources and microphones in the room: The loudspeaker at the center is the primary source, the other three loudspeakers are secondary sources, the microphones pairs labeled 1,$2 ; 3,4 ; 5,6 ; 7,8$ are the error sensors, and the microphones labeled $9,10,11, \ldots, 16$ monitor the noise pressure levels in the room. (b) A top view of the primary source $(\triangle)$, the secondary sources ( $(4))$ ), and the errors sensors $\circ$.

sound is $c \approx 343 \mathrm{~m} / \mathrm{s}$. The overall electrical delay of the ANC system is $5.3 \mathrm{~ms}$.

We conduct two hardware demonstrations. In the first demonstration, we use the secondary sources to control the noise produced by the primary source at a single frequency $f$, where $f=100,110, \ldots, 500 \mathrm{~Hz} .{ }^{31}$ Prior to the operation of the ANC system, we let each secondary source produces a noise of the form $\cos (2 \pi f t)$ over a period of $T=8 \mathrm{~s}$. Recordings from the four error sensors (the microphone pairs labeled 1,$2 ; 3,4 ; 5,6 ; 7,8)$ are transformed into the frequency-domain. Denote the frequency-domain noise pressures at a microphones pair be $P_{2 q-1}\left(k, R_{1}, \Theta_{q}\right)$ and $P_{2 q}\left(k, R_{2}, \Theta_{q}\right)$, where $q \in[1,4]$. We obtain the pressure and radial particle velocity at $\left(R, \Theta_{q}\right)$ through

$$
\begin{aligned}
& P_{q}\left(k, R, \Theta_{q}\right) \approx 0.5\left[P_{2 q}\left(k, R_{2}, \Theta_{q}\right)+P_{2 q-1}\left(k, R_{1}, \Theta_{q}\right)\right], \\
& V_{q}\left(k, R, \Theta_{q}\right) \approx \frac{i}{\rho c k} \frac{P_{2 q}\left(k, R_{2}, \Theta_{q}\right)-P_{2 q-1}\left(k, R_{1}, \Theta_{q}\right)}{\delta_{d}},
\end{aligned}
$$

where $\delta_{d}=R_{2}-R_{1}=0.1 \mathrm{~m}$. To use full spherical harmonics expansion, we mirror the pressure and radial particle velocity on the upper semi-sphere to the lower semi-sphere. ${ }^{17}$ We use the sound field separation method to obtain the outgoing field coefficients of the secondary sources, and these coefficients constitute the matrix $\boldsymbol{G}(k)$ in Eq. (15). We then let the primary source produce a unit-amplitude tonal noise and cancel the primary noise field in the room following the signal flow diagram as shown in Fig. 2.

In Fig. 11, we depict the noise energy reduction at the 16 microphones as $\xi(k)$, and

$$
\xi(k)=10 \log _{10} \frac{\sum_{v=1}^{16}\left\|P_{v}(k)\right\|^{2}}{\sum_{v=1}^{16}\left\|E_{v}(k)\right\|^{2}},
$$

where $P_{v}(k)$ and $E_{v}(k)$ are the primary and residual noise pressures at the $v$ th microphone, respectively. As shown in Fig. 11, we reduce the noise energy at the 16 microphones by more than $13 \mathrm{~dB}$ over the frequency range $[100,450] \mathrm{Hz}$. Measurements using a sound level meter reveal that the noise pressure levels are reduced by about $10 \mathrm{~dB}$ over the frequency range $[100,450] \mathrm{Hz}$ in the room.

The arrangement of the sources and error sensors in the first hardware demonstration is similar to case (2) in Sec. $\mathrm{VD}$ in the simulation. A comparison of Fig. 11 with $\xi_{2}(k)$ in Fig. 9 reveals that the energy reduction level in the hardware demonstration is about $5-8 \mathrm{~dB}$ less than in the simulation. This is because, as shown in Fig. 10, our lab is much more complicated than the simulation environment. Overall, the simulation and experimental results are consistent and demonstrate that the proposed ANC system can reduce outgoing noise fields over a wide frequency range.

In the second demonstration, the basic settings are the same as in the first demonstration, except that the primary noise consists of three unit-amplitude tonal waves of 150 , 200, and $310 \mathrm{~Hz}$. Denote the time domain primary noise pressure at the $q$ th microphone as $p_{q}(t)$, and the residual noise pressure at the microphone as $e_{q}(t)=p_{q}(t)+s_{q}(t)$, where $s_{q}(t)$ is the secondary noise pressure at that microphone. We record the primary noise pressures $\left\{p_{q}(t)\right\}_{q=1}^{16}$

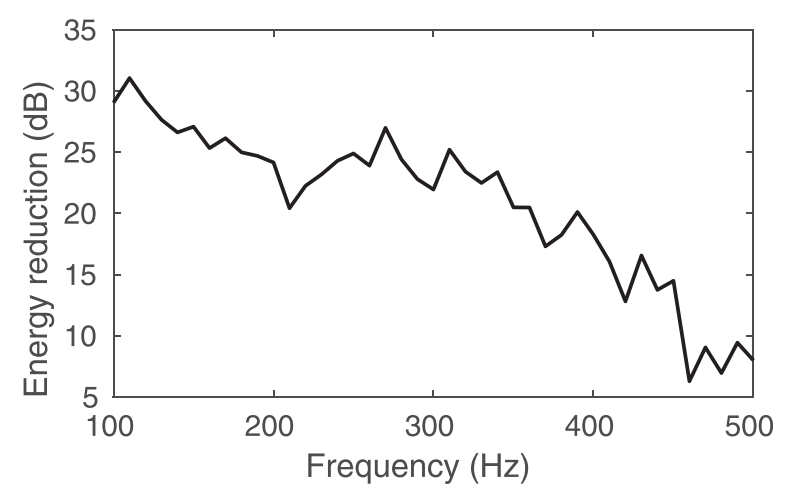

FIG. 11. Noise cancellation over a wide frequency range: Noise energy reduction $\xi(k)$ at the 16 microphones over the frequency range [100, 500] $\mathrm{Hz}$ using the demonstration hardware. 
TABLE I. Noise energy reduction $\left\{\xi_{q}\right\}_{q=1}^{16}$ at the 16 microphones over 20 seconds.

\begin{tabular}{lcccccccc}
\hline \hline$q$ & 1 & 2 & 3 & 4 & 5 & 6 & 7 & 8 \\
\hline$\xi_{q}(\mathrm{~dB})$ & 18.4 & 18.7 & 19.4 & 17.2 & 21.4 & 19.2 & 14.3 & 12.5 \\
$q$ & 9 & 10 & 11 & 12 & 13 & 14 & 15 & 16 \\
\hline$\xi_{q}(\mathrm{~dB})$ & 11.7 & 8.4 & 8.8 & 14.7 & 10 & 8.1 & 9.6 & 13.6 \\
\hline \hline
\end{tabular}

and the residual noise pressures $\left\{e_{q}(t)\right\}_{q=1}^{16}$ over $20 \mathrm{~s}$, respectively. Let the noise energy reduction at the microphones be defined as

$$
\xi_{q}=10 \log _{10} \frac{\sum_{t=t_{1}}^{T=t_{1}+20} p_{q}(t)^{2}}{\sum_{t=t_{2}}^{T=t_{2}+20} e_{q}(t)^{2}}, \quad q \in[1,16],
$$

where $t_{1}$ and $t_{2}$ are the time instants we start recording the primary noise pressures and the residual noise pressures, respectively. We present the noise energy reduction at the microphones in Table I. As shown in Table I, the proposed system reduces noise energy at the four microphone pairs (labeled as 1, 2; 3, 4; 5, 6; 7, 8 in Fig. 10) by more than $12.5 \mathrm{~dB}$, and at the eight monitoring microphones (labeled as $9,10, \ldots, 16$ in Fig. 10) by more than 8 dB. We have recorded the second demonstration in Mm. 1 .

Mm. 1. Recording of the second demonstration: We first let the primary source produce a tonal noise field in the room, then use the secondary outgoing field to destructively interfere with the primary outgoing field. The overall noise pressure level in the room is reduced by about $10 \mathrm{~dB}$. This is a file of type "mp4" $(9.9 \mathrm{Mb})$.

In all the simulations and hardware demonstrations, the proposed system reduces the noise fields globally in the entire room exterior to the semi-sphere $\mathbb{S}_{2}$ (the error microphones) without online estimation of the secondary paths.

\section{CONCLUSION}

This paper proposed an ANC system that can cancel tonal noise fields in rooms without the need for online secondary path estimation. The idea of the proposed ANC system is to cancel the outgoing field produced by the primary source on a small sphere surrounding the primary source, instead of controlling the noise pressure at multiple points ${ }^{3}$ or the room modes. ${ }^{5,6}$ By canceling the outgoing field only, the system's influence on the desired sound fields in the room is reduced.

\section{ACKNOWLEDGMENTS}

This work is sponsored by the Australian Research Council (ARC) Discovery Projects funding schemes with project Nos. DP140103412 and DP180102375. F.M. is supported by the China Scholarship Council-Australian
National University Joint Funding Program. We would like to thank Dr. Hanchi Chen for helping to design the hardware implementation of the system.

${ }^{1}$ S. M. Kuo and D. R. Morgan, "Active noise control: A tutorial review," Proc. IEEE 87(6), 943-973 (1999).

${ }^{2}$ S. J. Elliott, I. M. Stothers, and P. A. Nelson, "A multiple error LMS algorithm and its application to the active control of sound and vibration," IEEE Trans. Acoust. Speech Signal Process. 35(10), 1423-1434 (1987).

${ }^{3}$ C. Bao, P. Sas, and H. Van Brussel, "Adaptive active control of noise in 3D reverberant enclosures," J. Sound Vib. 161(3), 501-514 (1993).

${ }^{4}$ A. J. Kempton, "The ambiguity of acoustic sources-a possibility for active control?," J. Sound Vib. 48(4), 475-483 (1976).

${ }^{5}$ P. A. Nelson, A. R. D. Curtis, S. J. Elliott, and A. J. Bullmore, "The active minimization of harmonic enclosed sound fields, part i: Theory," J. Sound Vib. 117(1), 1-13 (1987).

${ }^{6}$ A. Montazeri, J. Poshtan, and M. H. Kahaei, "Modal analysis for global control of broadband noise in a rectangular enclosure," J. Low Freq. Noise Vib. Active Cont. 26(2), 91-104 (2007).

${ }^{7}$ T. Martin and A. Roure, "Optimization of an active noise control system using spherical harmonics expansion of the primary field," J. Sound Vib. 201(5), 577-593 (1997).

${ }^{8}$ B. Rafaely, "Spherical loudspeaker array for local active control of sound," J. Acoust. Soc. Am. 125(5), 3006-3017 (2009).

${ }^{9}$ E. G. Williams, Fourier Acoustics: Sound Radiation and Nearfield Acoustical Holography (Academic Press, New York, 1999), Chaps. 6 and 7, pp. 183-249.

${ }^{10} \mathrm{~T}$. Abhayapala, "Modal analysis and synthesis of broadband nearfield beamforming arrays," Ph.D. thesis, The Australian National University, 1999.

${ }^{11}$ H. Chen, T. D. Abhayapala, and W. Zhang, "Active noise control over space: A wave domain approach,” J. Acoust. Soc. Am. 138(5), 3081-3092 (2015).

${ }^{12}$ J. Zhang, T. D. Abhayapala, W. Zhang, P. N. Samarasinghe, and S. Jiang, "Active noise control over space: A wave domain approach," IEEE/ACM Trans. Audio Speech Lang. Process. 26(4), 774-786 (2018).

${ }^{13}$ Y. Huang, J. Benesty, and J. Chen, "Identification of acoustic mimo systems: Challenges and opportunities," Signal Process. 86(6), 1278-1295 (2006).

${ }^{14}$ A. Fahim, P. N. Samarasinghe, and T. D. Abhayapala, "Sound field separation in a mixed acoustic environment using a sparse array of higher order spherical microphones," in Proceedings of the Hands-free Speech Communications and Microphone Arrays (2017), pp. 151-155.

${ }^{15}$ A. J. Berkhout, D. de Vries, and P. Vogel, "Acoustic control by wave field synthesis," J. Acoust. Soc. Am. 93(5), 2764-2778 (1993).

${ }^{16}$ At low frequency range, where the wavelength of the noise is large compared with the size of the source, the scattered sound field is negligible (Ref. 17).

${ }^{17}$ M. Melon, C. Langrenne, P. Herzog, and A. Garcia, "Evaluation of a method for the measurement of subwoofers in usual rooms," J. Acoust. Soc. Am. 127(1), 256-263 (2010).

${ }^{18}$ D. B. Ward and T. D. Abhayapala, "Reproduction of a plane-wave sound field using an array of loudspeakers," IEEE Trans. Speech Audio Process. 9(6), 697-707 (2001).

${ }^{19}$ T. D. Abhayapala, T. S. Pollock, and R. A. Kennedy, "Characterization of 3D spatial wireless channels," IEEE 58th Vehicular Technology Conference (2003), Vol. 1, pp. 123-127.

${ }^{20}$ N. A. Gumerov and R. Duraiswami, Fast Multipole Methods for the Helmholtz Equation in Three Dimensions (Elsevier, London, 2005), pp. $1-520$.

${ }^{21}$ R. A. Kennedy, P. Sadeghi, T. D. Thushara, and H. M. Jones, "Intrinsic limits of dimensionality and richness in random multipath fields," IEEE Trans. Signal Process. 55(6), 2542-2556 (2007).

${ }^{22}$ W. Zhang, T. D. Thushara, R. A. Kenndy, and R. Duraiswami, "Insights into head-related transfer function: Spatial dimensionality and continuous representation," J. Acoust. Soc. Am. 127(4), 2347-2357 (2010).

${ }^{23}$ B. Rafaely, Fundamentals of Spherical Array Processing (Springer, New York, 2015), Chap. 3, pp. 57-78.

${ }^{24}$ X. Qiu, C. H. Hanson, and X. Li, "A comparison of near-field acoustic error sensing strategies for the active control of harmonic free field sound radiation," J. Sound Vib. 215(1), 81-103 (1998).

${ }^{25} \mathrm{~N}$. Han and X. Qiu, "A study of sound intensity control for active noise barriers,” Appl. Acoust. 68(10), 1297-1306 (2007). 
${ }^{26}$ In this paper, we have skipped the time index $t$ whenever possible to simplify the notations.

${ }^{27}$ H. Kuttruff, Room Acoustics (CRC Press, London, 2000), Chaps. 2-5, pp. 35-159.

${ }^{28}$ J. B. Allen and D. A. Berkley, "Image method for efficiently simulating small-room acoustics," J. Acoust. Soc. Am. 65(4), 943-950 (1979).

${ }^{29} \mathrm{P}$. A. Nelson and S. J. Elliott, The Active Control of Sound (Pearson Higher Ed, New York, 1991), Chaps. 10-12, pp. 310-410.
${ }^{30} \mathrm{We}$ can use a microphone pair as the error sensor as shown in Sec. VI. The microphone pair can measure the noise pressure at two points. To make the comparison fair, we design the classical multichannel ANC system to cancel the noise pressure at $4 \times 2$ points.

${ }^{31}$ Due to their limited volume, the loudspeakers are unable to radiate very low frequency $(f<100 \mathrm{~Hz})$ sound effectively. Thus the demonstrations are conducted for higher frequency, where $100<f$ $<500 \mathrm{~Hz}$. 